\title{
Faecal stasis in proctocolitis
}

\author{
J. E. LENNARD-JONES, M. J. S. LANGMAN, AND F. AVERY JONES \\ From St. Mark's Hospital, London, and the Department of Gastroenterology, \\ Central Middlesex Hospital, London
}

EDITORIAL SYNOPSIS Six cases of faecal stasis in active proctocolitis are reported. In each case the left colon was severely diseased but the right colon was unaffected. $X$-ray studies showed retention of barium in the right colon for as long as five weeks. In two patients subacute intestinal obstruction developed and in three patients constipation was a major symptom during the active phase of colitis.

Usually a patient with colitis passes liquid stools, but in certain patients with colitis the liquid passed consists almost wholly of blood and pus and the faeces passed are solid. We report here six patients who passed liquid stools but in whom there was considerable faecal stasis in the proximal colon. This was sufficient to produce symptoms in four patients, and paradoxically these patients with active colitis needed treatment with laxatives. In cases 1 and 2 faecal impaction in the right colon produced acute abdominal tenderness and subacute intestinal obstruction. In cases 2,3 , and 4 a sense of constipation and the passage of hard stools were major symptoms. In cases 5 and 6 marked faecal stasis was present in the right colon but its clinical significance was doubtful.

\section{CASE REPORTS}

CASE 1 Four months before admission, after an episode of constipation, this woman, aged 32, noticed rectal bleeding. Diarrhoea began and increased until she was passing six or seven loose, blood-stained stools daily. She felt ill, lost 1 stone in weight and, two weeks before admission, began to vomit. At the time of admission she was febrile, lesions of erythema nodosum were present on both legs, and sigmoidoscopy showed typical changes of active colitis. A barium enema showed gross changes of proctocolitis with apparent deep ulceration affecting the colon distal to the splenic flexure. She was treated with oral prednisone, sulphasalazine, and retention enemas of hydrocortisone.

One week after admission a mass was noted in the right iliac fossa and was palpable for over three weeks. During this time she passed on average five loose stools daily, the number slowly decreasing as time passed. Four weeks after admission, and five weeks after the barium enema, severe griping abdominal pain developed associated with vomiting and acute tenderness and rigidity in the right iliac fossa. The mass was still palpable despite the rigidity and the diagnoses considered were faecal impaction with subacute intestinal obstruction, stercoral ulceration with pericolic inflammation, localized perforation of the colon and pericolic abscess, or acute appendicitis. A radiograph of the abdomen showed a dense mass of inspissated barium about 2 in. in diameter (Fig. 1) in the region of the hepatic flexure with accumu-

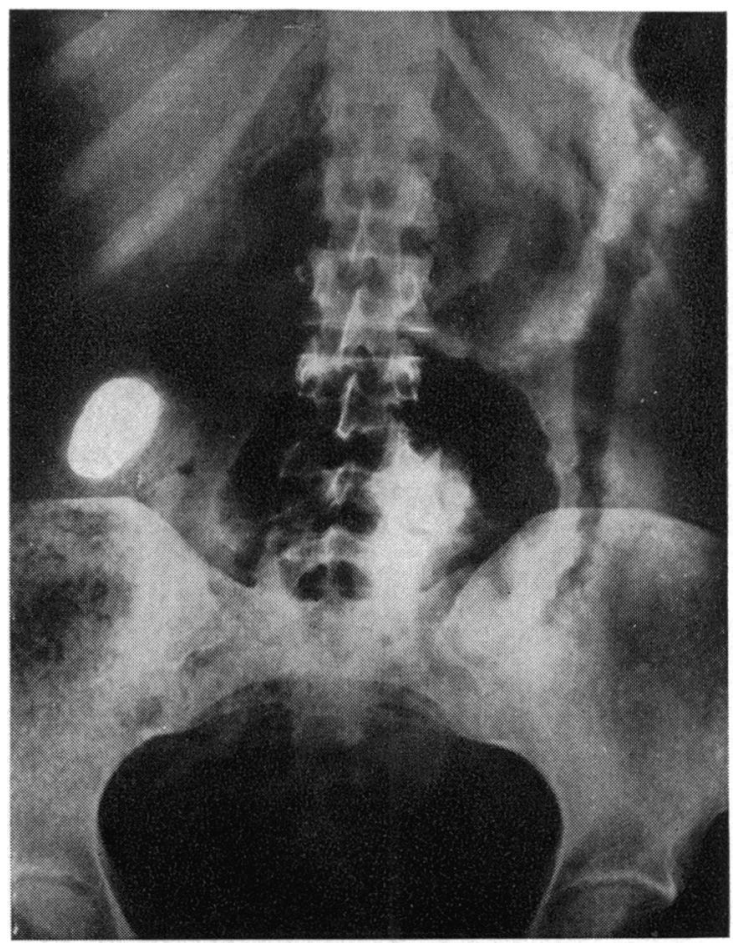

FIG. 1. Case 1: Barium mass remaining in the ascending colon after a barium enema five weeks before: the high density of the mass suggests absorption of water from the barium suspension. Faecal material is layered around the barium concretion and retained behind it. The air outline of the left colon shows that it is narrow and irregular. The barium enema had shown severe ulceration of this part of the colon. 
lated faecal material apparently layered around it and retained behind it. The ascending colon and caecum were distended with faeces and the lower ileum was distended with gas. The distal colon, outlined by gas in the region of the transverse colon and splenic flexure, showed irregularity and constriction of its lumen. Subacute intestinal obstruction due to impacted barium and faeces in the ascending colon was diagnosed. Magnesium sulphate was given by mouth, a large faecal mass was passed and then the pain and tenderness subsided. A further radiograph showed that the mass of barium was no longer present though some faecal material remained in the ascending colon. She was discharged from hospital three weeks later free of symptoms. When last seen, one month later, she felt well, was passing one formed stool daily and was about to return to work.

CASE 2 At the age of 47 this patient experienced a short episode of diarrhoea with rectal bleeding. Eight years later she was referred to hospital because she had become constipated and had passed blood per rectum. Sigmoidoscopy revealed active mucosal inflammation extending for $15 \mathrm{~cm}$. above the anus; beyond this distance the colonic mucosa appeared normal. She was admitted to hospital two months later because she became severely ill, with fever, constant nausea, weight loss, and the passage of several dark red, liquid stools each day. On examination she was pale ( $\mathrm{Hb} 36 \%$ ), the abdomen was distended, and a large mass could be felt in the right iliac fossa. Sigmoidoscopy to $13 \mathrm{~cm}$. revealed an inflamed rectal mucosa, the lumen containing blood and mucus. Perforation of the colon and a pericolic abscess were suspected. However, radiographs of the abdomen showed no abnormality other than distension of the caecum by faeces. A few days later the patient had a rigor with a fever of $103^{\circ} \mathrm{F}$. and at this time constipated stools were passed. A barium enema showed a somewhat dilated but otherwise normal colon containing many faecal masses. Constipation remained a problem until she was discharged from hospital free of symptoms after six weeks' treatment with corticosteroids, frequent olive oil enemas, and oral laxatives.

After two weeks she was re-admitted to hospital because of severe abdominal pain. She again looked ill, the descending colon was palpable and heavily loaded; the ascending colon felt distended and was tender. On sigmoidoscopy, the rectum appeared very inflamed and many pseudo-polypi were seen. That evening she passed a large stool, her condition improved, and the colon became impalpable. During the next five days no bowel action occurred though blood was frequently passed. She suddenly deteriorated with nausea, a rigor, and abdominal distension two weeks later, despite treatment with prednisone and regular doses of magnesium hydroxide with liquid paraffin emulsion. A radiograph of the abdomen showed that the whole colon was distended with faeces and some fluid levels were present in the small intestine. Daily saline washouts were instituted. Some days later she had a severe rectal haemorrhage, necessitating immediate transfusion, and haemorrhage continued intermittently for some days. Surgical treatment was decided upon and subtotal colectomy with ileostomy performed.

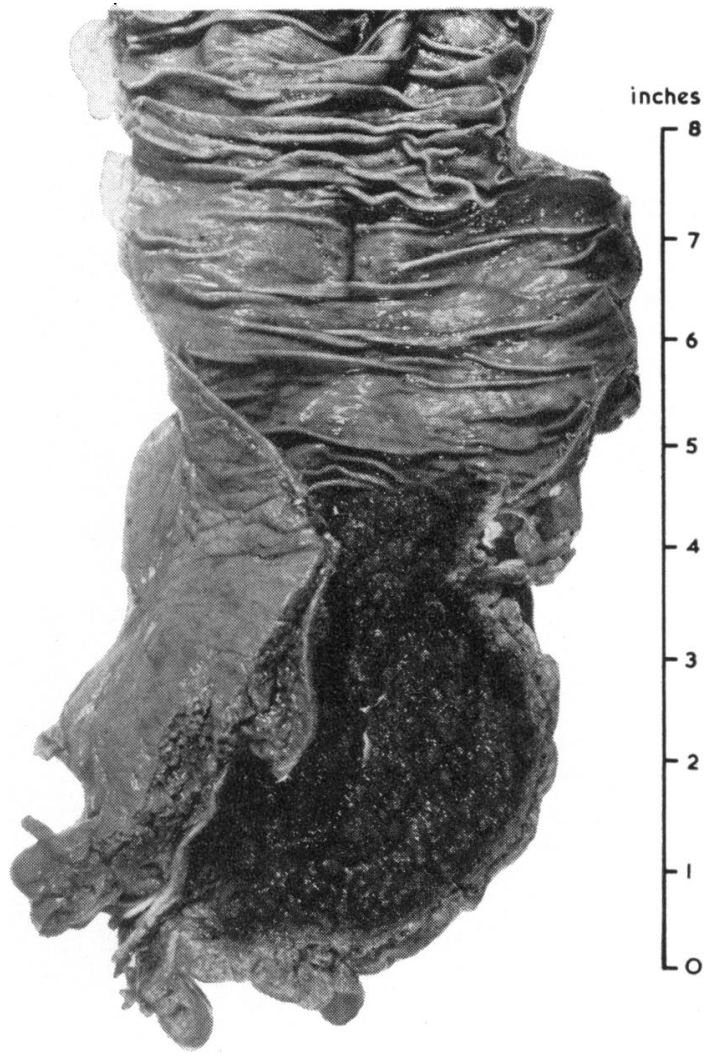

FIG. 2. Case 2: Junction of normal and severely diseased mucosa in the distal sigmoid colon. The colon was normal proximal to this level.

The operation specimen (Fig. 2) showed a normal but dilated colon proximal to an area of severe disease with ulceration, crypt abscesses, and numerous pseudopolypi, involving the distal sigmoid colon and rectum. Recovery was delayed by a lung abscess but the patient is now well.

CASE 3 A woman of 35 was admitted to hospital with a four-month history of passing blood and pus per rectum. The symptoms had not improved during out-patient treatment with prednisone and sulphasalazine. In hospital she complained bitterly of a sense of constipation; she passed small quantities of blood several times a day and a small constipated stool every few days. On palpation of the abdomen the ascending colon felt distended and on sigmoidoscopy the rectal mucosa was actively inflamed but the rectum was empty. A barium enema (Fig. 3) showed severe changes of colitis in the distal colon, with narrowing and pseudo-polypi, and distension of the proximal colon, which appeared free of disease but contained lumps of solid faeces. The colitis was treated with A.C.T.H., hydrocortisone retention enemas, and large doses of magnesium hydroxide emulsion with liquid paraffin by mouth. Her condition slowly improved 


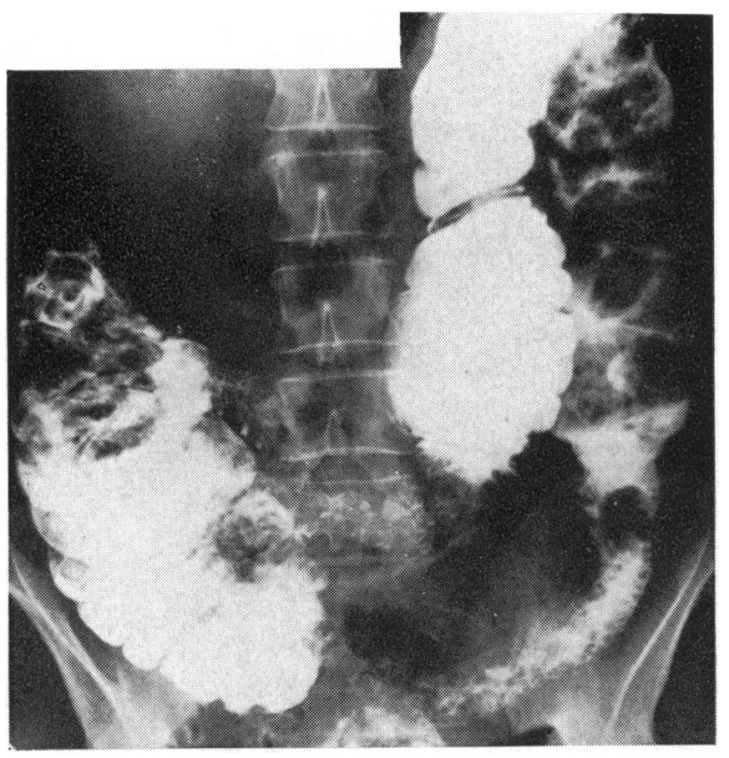

FIG. 3. Case 3: The lower part of the descending colon is severely narrowed with pseudo-polypoid changes. Many solid faecal masses are present in the dilated colon above the diseased area.

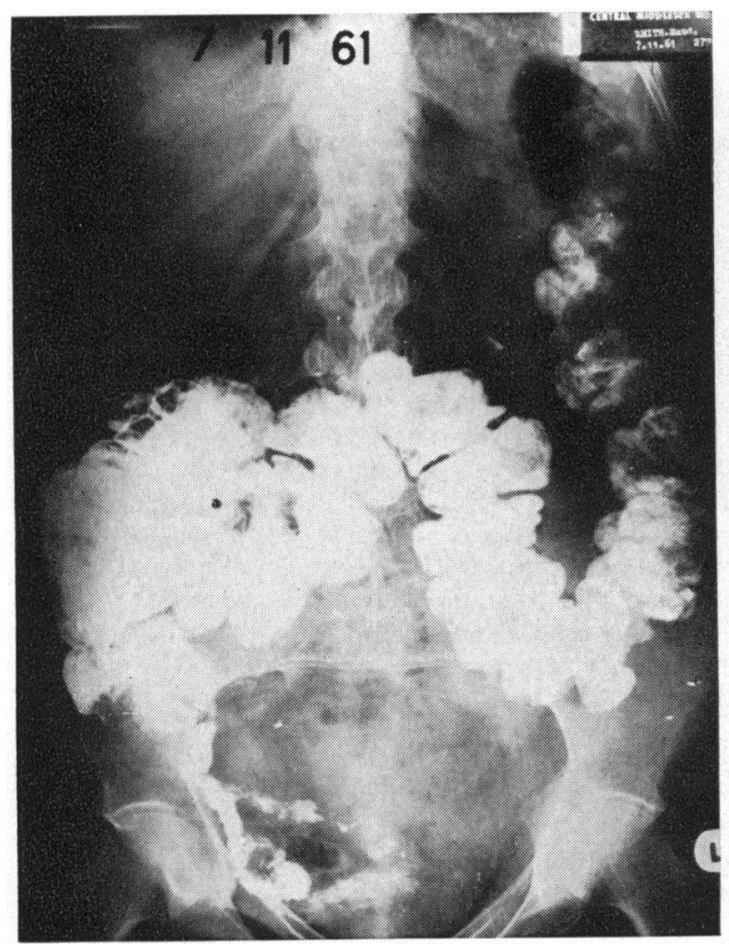

FIG. 4a. and the colonic distension subsided. When last seen, six months after leaving hospital, she was well and passing one normal motion daily with a little blood and mucus.

CASE 4 This woman of 70 was admitted to hospital with a six-month history of increasing rectal bleeding, diarrhoea, weight loss, and latterly of high fever. For several days after admission she ran a remittent fever of 102 to $103^{\circ} \mathrm{F}$. On sigmoidoscopy, the colonic mucosa was seen to be actively inflamed. A barium enema showed apparent deep ulceration involving the descending colon; the colon above this level could not be filled. She was given A.C.T.H. and her condition slowly improved, the initial diarrhoea being replaced by severe constipation; indeed, a second barium enema examination performed two months after admission was technically unsatisfactory because of impacted faecal masses in the ascending colon. The radiologist commented that these masses must have taken weeks to accumulate. Treatment of the constipation with oral laxatives and olive oil enemas became a major problem in the management of the illness. After the faecal masses were passed, constipation ceased to be a problem and the patient was discharged home free of symptoms six weeks after admission to hospital.

A relapse of the disease occurred just over a year later. Before treatment on this occasion, she was passing nine to 12 bowel motions daily, a soft motion in the morning and thereafter loose stools containing much mucus. One

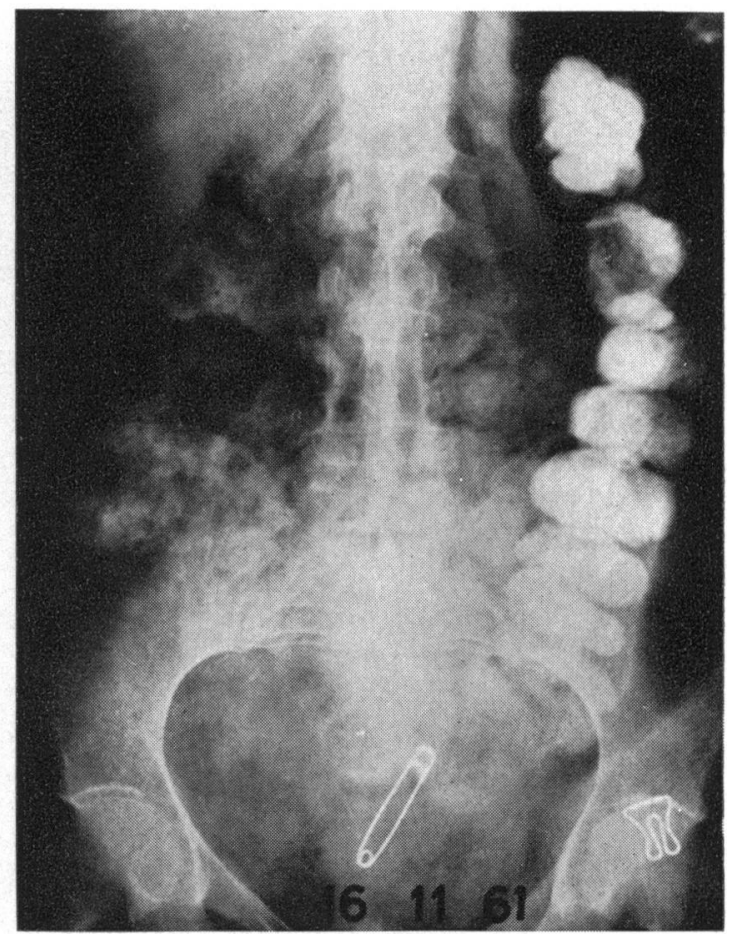

FIG. 4b. 


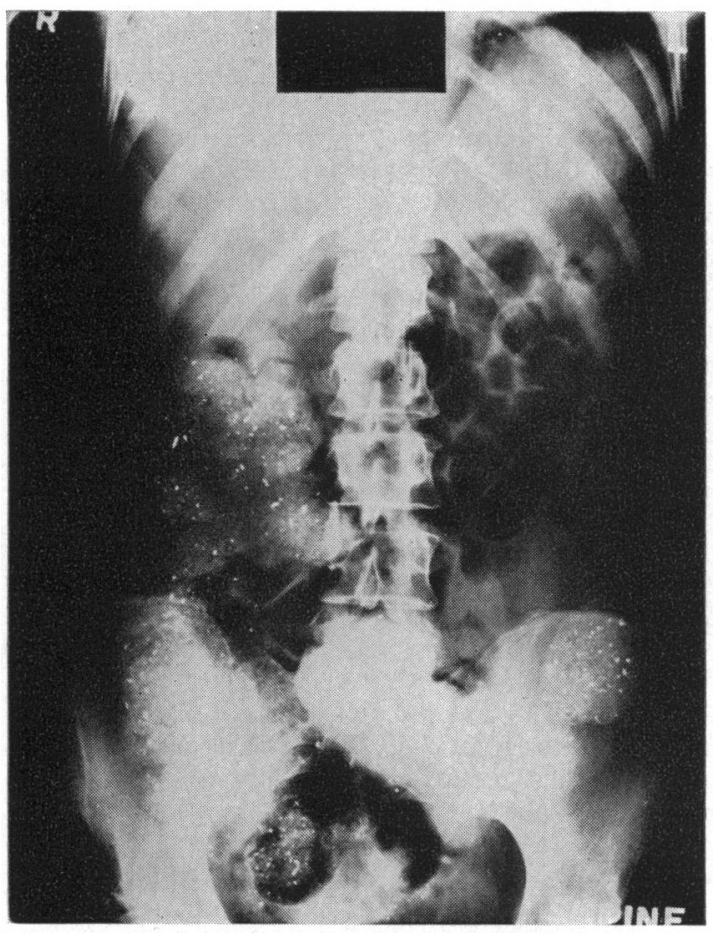

FIG. 5a.

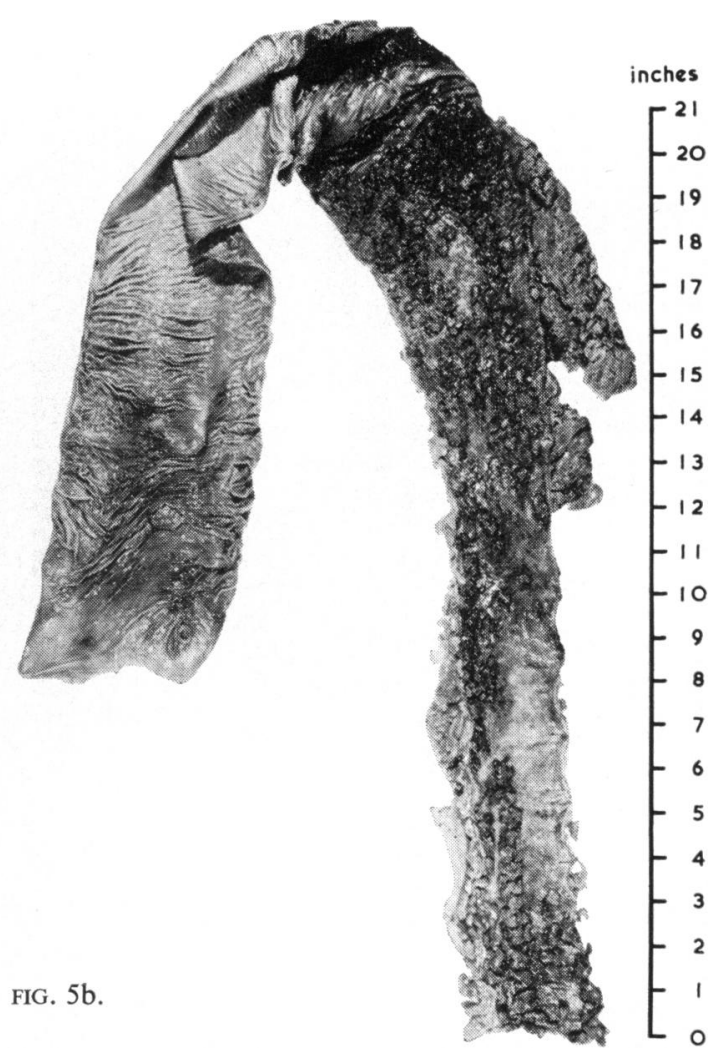

FIG. 5. Case 5: (a) Barium administered four weeks previously as an enema is retained within faecal masses in the right and descending colon. (b) Operation specimen showing that the colon was almost normal proximal to the mid-transverse colon, but distally there was severe disease with extensive stripping of the mucosa leaving isolated mucosal tags.

week after starting treatment with prednisone the number fell to three formed stools daily. Abdominal distension now developed and the stools became hard and infrequent, though blood-stained mucus was passed frequently. A radiograph of the abdomen showed distension of the proximal colon with gas and faeces. Abdominal distension became the main symptom and this was apparently associated with faecal stasis, the hard, loaded ascending colon being easily palpable. A small quantity of barium suspension was given by mouth and its progress followed by daily radiographs. These films showed that within 24 hours the barium had reached the right colon (Fig. 4a), but thereafter progress was so slow that nine days later the descending colon was still outlined (Fig. 4b). She was treated with prednisone, sulphasalazine, magnesium hydroxide, liquid paraffin and methyl cellulose by mouth, and by olive oil enemas. The distension and faecal stasis gradually resolved and she was discharged well five weeks after admission.

CASE 5 This patient, aged 51, was admitted to hospital elsewhere with a short history of severe diarrhoea and systemic illness. There was no response to treatment with cortisone and sulphasalazine and, as her condition gave rise to anxiety, she was transferred after two months to St. Mark's Hospital for surgical treatment. When first seen, she was pale, lethargic, vomiting frequently, and passing about eight liquid stools each day. There was moderate fever; the abdomen was slightly distended but no masses were palpable. A radiograph of the abdomen showed an unexpected degree of faecal stasis because barium, introduced exactly one month previously, was still present mixed with faecal material in the caecum (Fig. 5a). Faecal masses persisted in the caecum for almost two weeks despite colonic wash-outs. For two weeks the patient's condition gradually improved with supportive treatment, but then deteriorated suddenly with increased fever and abdominal tenderness.

Subtotal colectomy with ileostomy was performed, and the post-operative recovery was uneventful. The operation specimen (Fig. 5b) showed that the caecum and proximal half of the transverse colon were almost normal but distally there was extensive stripping of mucosa, leaving mucosal tags. Histologically, the inflammatory process was seen to penetrate all layers of the bowel wall in the sigmoid colon.

CASE 6 This man, aged 54, was admitted to hospital three months after developing diarrhoea. During the week before admission he lost all desire for food, his abdomen became distended, and he passed about 10 motions daily consisting mainly of blood. He looked ill, was running a 
fever up to $102^{\circ} \mathrm{F}$., the abdomen was distended and tender, and on sigmoidoscopy the rectal mucosa appeared actively inflamed. Two days later he was worse and had 20 bowel actions during one night. On this day radiography of the abdomen showed the presence of much faecal material in the ascending colon. Treatment with A.C.T.H. resulted in immediate improvement but some fever persisted and several liquid, brown, blood-stained stools were passed each day. A further radiograph of the abdomen three weeks after admission still showed faecal masses in the right colon. Four days later a mouthful of barium suspension was given. Daily radiographs showed that the barium remained in the caecum for six days though liquid stools were still being passed. An oral dose of $\mathrm{Cr}^{51}$ as sodium chromate, an unabsorbed tracer, was administered, and in $\mathbf{4 8}$ hours, despite the passage of seven liquid stools, only $12 \%$ of the dose was excreted as compared to a mean normal excretion of $54.3 \%$ (Hansky and Connell, 1962). Oral sodium sulphasuccinate was added to the treatment régime. Over the next few days the fever subsided, his appetite returned, and one week later a formed stool was passed for the first time since the illness began. A barium enema at this stage showed an apparently normal ascending and proximal transverse colon. Distal to the mid-transverse colon, the bowel was shortened and narrowed; numerous mucosal filling defects were apparent in the descending colon. The patient was discharged, feeling well and passing two semi-formed stools daily, seven weeks after admission.

\section{DISCUSSION}

Certain features were common to these six patients. All had severe ulcerative colitis of part or all of the left colon and in all of them the right colon was apparently normal. All passed frequent liquid stools at a time when the right colon was filled with faecal material. In four of the six patients there was radiological evidence of prolonged retention of barium suspension in the right colon, in one case for four and in another for five weeks. It seems possible that the severe colitis in the left colon acted as an obstruction to the passage of solid faeces while at the same time the water-absorptive capacity of the normal right colon remained unimpaired so that inspissation of the contents of the right colon followed. The high density of the barium concentration in case 1 (Fig. 1) suggests that water was absorbed in the ascending colon of this patient. The slow passage of the unabsorbed tracer in case 5 suggests that the frequent liquid stools passed by these patients consist mainly of exudate from the diseased colon.

In five of these patients faecal stasis was an unexpected finding on a plain radiograph of the abdomen. This is only part of the information gained from such a film which can be helpful in assessing the state of the colon in patients with colitis. A plain radiograph has revealed unexpected faecal stasis in other patients with colitis but we have not assessed its frequency and do not know how often it is of clinical significance.

If faecal stasis is thought to be aggravating the condition of a patient with colitis specific treatment is indicated. Opiates and anticholinergic drugs should be stopped and then, if necessary, oral laxatives should be given. Saline purges, liquid paraffin and wetting agents, such as dioctyl sodium sulphasuccinate, may be helpful. Saline wash-outs are probably ineffective when the faecal accumulation is in the right colon but retention enemas of olive oil seem beneficial when hard faeces in the rectum are causing discomfort.

There is little comment in the literature about faecal stasis in colitis, though constipation occurs in proctitis (Lennard-Jones, Cooper, Newell, Wilson, and Avery Jones, 1962). Henderson (1954) suggested that constipation may be followed by colitis, and introduced the concept of faecal stasis at St. Mark's Hospital by describing cases similar to those reported here as suffering from 'proximal constipation and distal diarrhoea'. The term 'faecal stasis' seems preferable as it is free of other connotation.

We thank Dr. Richard Asher (case 1), Mr. O. V. Lloyd Davies (cases 2 and 4), and Mr. C. Naunton Morgan (case 5) for permission to report their cases. We thank Dr. J. Hansky for the radio-isotope study in case 6 and the $X$-ray and Photographic Departments at the Central Middlesex and St. Mark's Hospitals for the illustrations.

\section{REFERENCES}

Hansky, J., and Connell, A. M. (1962). Measurement of gastrointestinal transit using radioactive chromium. Gut, 3, 187-188.

Henderson, N. P. (1954). What is ulcerative colitis? Lancet, 1, 159-160. Lennard-Jones, J. E., Cooper, G. W., Newell, A. C., Wilson, C. W. E., and Jones, F. Avery (1962). Observations on idiopathic proctitis. Gut, 3, 201-206. 